\title{
Transverse momentum broadening of hadrons produced in semi-inclusive deep-inelastic scattering on nuclei
}

\section{Hermes Collaboration}
A. Airapetian ${ }^{1,0}$, N. Akopov ${ }^{\mathrm{z}}$, Z. Akopov ${ }^{\mathrm{e}}$, E.C. Aschenauer ${ }^{\mathrm{f}}, \mathrm{W}$. Augustyniak ${ }^{\mathrm{y}}$, A. Avetissian $^{\mathrm{z}}$, E. Avetisyan $^{\mathrm{e}}$, B. Ball ${ }^{\circ}$, S. Belostotski ${ }^{\mathrm{r}}$, N. Bianchi ${ }^{\mathrm{j}}$, H.P. Blok ${ }^{\mathrm{q}, \mathrm{x}}$, H. Böttcher $^{\mathrm{f}}$, C. Bonomo $^{\mathrm{i}}$, A. Borissov $^{\mathrm{e}}$, V. Bryzgalov ${ }^{\text {s }}$, J. Burns ${ }^{\mathrm{m}}$, M. Capiluppi ${ }^{\mathrm{i}}$, G.P. Capitani ${ }^{\mathrm{j}}$, E. Cisbani ${ }^{\mathrm{u}}$, G. Ciullo ${ }^{\mathrm{i}}$, M. Contalbrigo ${ }^{\mathrm{i}}$, P.F. Dalpiaz ${ }^{i}$, W. Deconinck ${ }^{e}, o$, L. De Nardo ${ }^{e},{ }$, R. De Leo $^{b}$, J. Dreschler ${ }^{q}$, E. De Sanctis ${ }^{j}$, M. Diefenthaler ${ }^{\text {n,h }}$, P. Di Nezza ${ }^{j}$, M. Düren ${ }^{1}$, M. Ehrenfried ${ }^{1}$, G. Elbakian ${ }^{z}$, F. Ellinghaus ${ }^{d}$, R. Fabbrif ${ }^{\text {, }}$, L. Felawka ${ }^{\text {v }, ~ A . ~ F a n t o n i ~}{ }^{j}$, S. Frullani ${ }^{\text {u }}$, D. Gabbert ${ }^{f}$, V. Gapienko ${ }^{\text {s }}$, F. Garibaldi ${ }^{\mathrm{u}}$, V. Gharibyan ${ }^{\mathrm{z}}$, F. Giordano ${ }^{\mathrm{e}, \mathrm{i}}$, S. Gliske $^{\mathrm{o}}$, C. Hadjidakis $^{\mathrm{j}}$, M. Hartig ${ }^{\mathrm{e}}$, D. Hasch ${ }^{\mathrm{j}}$, G. Hill ${ }^{\mathrm{m}}$, A. Hillenbrand ${ }^{\mathrm{f}}$, M. Hoek ${ }^{\mathrm{m}}$, Y. Holler $^{\text {e }}$, I. Hristova ${ }^{f}$, Y. Imazu ${ }^{\text {w }}$, A. Ivanilov ${ }^{\text {s }}$, H.E. Jackson ${ }^{\text {a }}$, H.S. Jo ${ }^{\mathrm{k}}$, A. Jgoun $^{\mathrm{r}}$, S. Joosten ${ }^{\mathrm{n}}$, R. Kaiser ${ }^{m}$, G. Karyan ${ }^{z}$, T. Keri ${ }^{m, 1}$, E. Kinney ${ }^{d}$, A. Kisselev ${ }^{r}$, V. Korotkov ${ }^{s}$, V. Kozlov ${ }^{\text {P }}$, P. Kravchenko ${ }^{r}$, L. Lagamba $^{b}$, R. Lamb ${ }^{n}$, L. Lapikás ${ }^{\text {, I. Lehmann }}{ }^{m}$, P. Lenisa ${ }^{\mathrm{i}}$, L.A. Linden-Levy ${ }^{\mathrm{n}}$, A. López Ruiz ${ }^{\mathrm{k}}$, W. Lorenzon ${ }^{\mathrm{O}}$, X.-G. Lu ${ }^{\mathrm{f}}$, X.-R. Lu ${ }^{\mathrm{w}}$, B.-Q. Ma ${ }^{\mathrm{c}}$, D. Mahon ${ }^{\mathrm{m}}$, N.C.R. Makins ${ }^{\mathrm{n}}$, S.I. Manaenkov ${ }^{\mathrm{r}}$, L. Manfré ${ }^{\mathrm{u}}$, Y. Mao ${ }^{\text {, }}$, B. Marianski ${ }^{\mathrm{y}}, \mathrm{A}$. Martinez de la Ossa ${ }^{\mathrm{d}}$, H. Marukyan ${ }^{\mathrm{z}}$, C.A. Miller ${ }^{\mathrm{v}}$, Y. Miyachi ${ }^{\mathrm{w}}$,

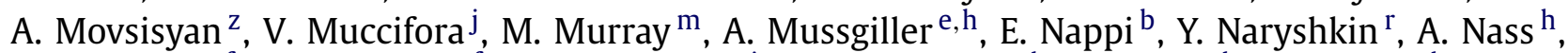 M. Negodaev ${ }^{\text {f, W.-D. Nowak }}{ }^{\text {, }}$, L.L. Pappalardo ${ }^{\mathrm{i}}$, R. Perez-Benito ${ }^{1}$, N. Pickert ${ }^{\mathrm{h}}$, M. Raithel ${ }^{\mathrm{h}}$, P.E. Reimer ${ }^{\mathrm{a}}$, A.R. Reolon ${ }^{\mathrm{j}}$, C. Riedl ${ }^{\mathrm{f}}$, K. Rith $^{\mathrm{h}, *}$, G. Rosner ${ }^{\mathrm{m}}$, A. Rostomyan $^{\mathrm{e}}$, J. Rubin ${ }^{\mathrm{n}}$, D. Ryckbosch ${ }^{\mathrm{k}}$, Y. Salomatin ${ }^{\mathrm{s}}$, F. Sanftl ${ }^{t}$, A. Schäfer ${ }^{t}$, G. Schnell ${ }^{f, k}$, K.P. Schüler ${ }^{\mathrm{e}}$, B. Seitz ${ }^{\mathrm{m}}$, T.A. Shibata ${ }^{\mathrm{w}}$, V. Shutov ${ }^{\mathrm{g}}$, M. Stancari ${ }^{\mathrm{i}}$, M. Statera ${ }^{\mathrm{i}}$, E. Steffens ${ }^{\mathrm{h}}$, J.J.M. Steijger ${ }^{\mathrm{q}}$, H. Stenzel ${ }^{1}$, J. Stewart ${ }^{\mathrm{f}}$, F. Stinzing ${ }^{\mathrm{h}}$, S. Taroian ${ }^{\mathrm{z}}$, A. Trzcinski ${ }^{\mathrm{y}}$, M. Tytgat ${ }^{\mathrm{k}}$, A. Vandenbroucke ${ }^{\mathrm{k}}$, P.B. van der Nat ${ }^{\mathrm{q}}$, Y. Van Haarlem ${ }^{\mathrm{k}}$, C. Van Hulse ${ }^{\mathrm{k}}$, D. Veretennikov ${ }^{\mathrm{r}}$,

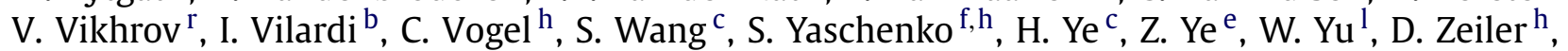 B. Zihlmann ${ }^{\text {e }}$, P. Zupranski ${ }^{y}$

\footnotetext{
a Physics Division, Argonne National Laboratory, Argonne, IL 60439-4843, USA

${ }^{\mathrm{b}}$ Istituto Nazionale di Fisica Nucleare, Sezione di Bari, 70124 Bari, Italy

c School of Physics, Peking University, Beijing 100871, China

d Nuclear Physics Laboratory, University of Colorado, Boulder, CO 80309-0390, USA

e DESY, 22603 Hamburg, Germany

${ }^{f}$ DESY, 15738 Zeuthen, Germany

goint Institute for Nuclear Research, 141980 Dubna, Russia

h Physikalisches Institut, Universität Erlangen-Nürnberg, 91058 Erlangen, Germany

i Istituto Nazionale di Fisica Nucleare, Sezione di Ferrara and Dipartimento di Fisica, Università di Ferrara, 44100 Ferrara, Italy

j Istituto Nazionale di Fisica Nucleare, Laboratori Nazionali di Frascati, 00044 Frascati, Italy

${ }^{\mathrm{k}}$ Department of Subatomic and Radiation Physics, University of Gent, 9000 Gent, Belgium

${ }^{1}$ Physikalisches Institut, Universität Gießen, 35392 Gießen, Germany

$\mathrm{m}$ Department of Physics and Astronomy, University of Glasgow, Glasgow G12 8QQ United Kingdom

${ }^{n}$ Department of Physics, University of Illinois, Urbana, IL 61801-3080, USA

${ }^{\circ}$ Randall Laboratory of Physics, University of Michigan, Ann Arbor, MI 48109-1040, USA

p Lebedev Physical Institute, 117924 Moscow, Russia

${ }^{\mathrm{q}}$ National Institute for Subatomic Physics (Nikhef), 1009 DB Amsterdam, The Netherlands

${ }^{\mathrm{r}}$ Petersburg Nuclear Physics Institute, Gatchina, Leningrad region 188300, Russia

${ }^{s}$ Institute for High Energy Physics, Protvino, Moscow region 142281, Russia

${ }^{\mathrm{t}}$ Institut für Theoretische Physik, Universität Regensburg, 93040 Regensburg, Germany
}

\footnotetext{
* Corresponding author.

E-mail address: klaus.rith@desy.de (K. Rith).
} 


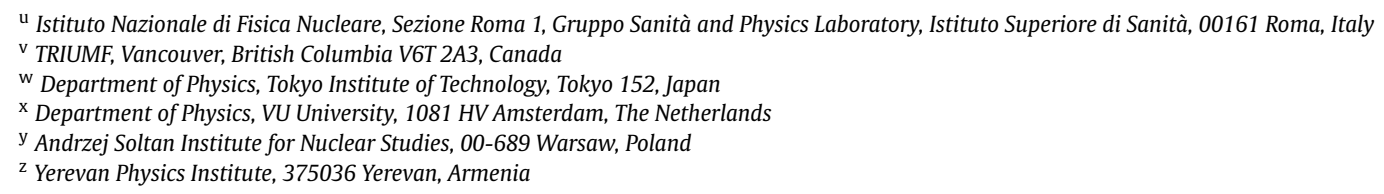

\section{A R T I C L E I N F O}

\section{Article history:}

Received 14 June 2009

Received in revised form 24 November 2009

Accepted 11 January 2010

Editor: V. Metag

\begin{abstract}
A B S T R A C T
The first detailed measurement of the dependence on target nuclear mass of the average squared transverse momentum $\left\langle p_{t}^{2}\right\rangle$ of $\pi^{+}, \pi^{-}$, and $K^{+}$mesons from deep-inelastic lepton scattering is obtained as a function of several kinematic variables. The data were accumulated at the HERMES experiment at DESY, in which the HERA $27.6 \mathrm{GeV}$ lepton beam was scattered off several nuclear gas targets. The average squared transverse momentum was clearly observed to increase with atomic mass number. The effect increases as a function of $Q^{2}$ and $x$ and remains constant as a function of both the virtual photon energy $v$ and the fractional hadron energy $z$, except that it vanishes as $z$ approaches unity.
\end{abstract}

(C) 2010 Elsevier B.V. All rights reserved.
The evolution of a fast-moving quark into hadrons is a nonperturbative and dynamic phenomenon. The basic process of hadronization in vacuum is described by a well-developed phenomenology, constrained primarily by data from inclusive hadron production in $e^{+} e^{-}$annihilation, semi-inclusive hadron multiplicities measured in deep-inelastic scattering (DIS) of leptons on protons and deuterons, and inclusive hadron yields from $\mathrm{p}-\mathrm{p}$ scattering experiments. For a recent global analysis of such data, see Ref. [1] and references therein.

The nuclear modification of hadron production in DIS was first observed by the pioneering semi-inclusive DIS experiments at SLAC [2], followed by measurements performed by the EMc [3] and the E665 [4] Collaboration. These experiments typically determined the ratio of hadron multiplicities observed in the scattering on a nucleus to those on deuterium (D), the so-called nuclear attenuation. More recently, much more precise data were collected and analyzed by the Hermes Collaboration $[5,6]$ as a function of the kinematic variables $v, z, Q^{2}$, and $p_{t}^{2}$, where $v$ is the energy of the virtual photon, $z$ the fractional hadron energy in the target rest frame, $-Q^{2}$ being the squared four-momentum of the virtual photon, and $p_{t}$ the transverse momentum of the produced hadron. It has been suggested [7-9] that hadron production proceeds through three qualitatively distinct stages that involve the propagation and interaction of:

(i) the initial struck quark ${ }^{1}$ (the "partonic" stage),

(ii) the subsequently formed colorless state (the "color-neutralization" stage, often also termed "pre-hadronic" stage), and

(iii) the final produced hadrons (the "hadronic" stage).

This picture is supported by the results of a two-dimensional analysis of the multiplicity ratio [6] in the kinematic variables $v$ and $z$. However, the existence and relative importance of the various stages to the observed nuclear attenuation have been difficult to determine unambiguously. This Letter reports, complimentary to the results presented in Ref. [6], the first detailed measurement of another observable, which may help to better constrain models, especially with regard to the role of the "partonic" stage: the broadening of the transverse momentum distribution of various hadrons.

In terms of the quark-parton model and QCD, there are several contributions to the transverse momentum distribution of hadrons produced in semi-inclusive DIS:

\footnotetext{
1 "quark" is used in this Letter for both quarks and antiquarks.
}

(a) primordial transverse momentum,

(b) gluon radiation of the struck quark,

(c) the formation and soft multiple interactions of the "prehadron", and

(d) the interaction of the formed hadrons with the surrounding hadronic medium.

In semi-inclusive DIS off nuclear targets, the struck quark propagates through a "cold" nuclear medium. In the nuclear medium the primordial transverse momentum of quarks may be modified by various effects like Fermi motion of nucleons inside the nucleus, modification of the nucleon size, formation of non-nucleonic degrees of freedom like multiquark states or exchange mesons mediating the nuclear force. Also, the probabilities of the processes (b-d) may be enhanced resulting in a larger transverse momentum magnitude of the observed hadrons relative to the process in the vacuum or in a free nucleon. In particular, process (b) may cause an increased transverse momentum magnitude and energy loss of the quark and therefore it has recently been suggested [10] that the broadening of hadron distributions in semi-inclusive DIS may be the most direct way to probe the "partonic" stage. Both the radiative energy loss and the transverse momentum of the parton increase with $L$, which is the path length of the quark in the nuclear medium before the colorless state formation. QCD predictions for the relationship between energy loss and the broadening of $p_{t}$ distributions are given in Refs. [11,12]. As the relationship between these two quantities is independent of the dynamics of the initial scattering process, it holds equally well in "cold" nuclear matter and in finite-length "hot" matter, produced, e.g., in ultrarelativistic heavy-ion collisions or high-energy proton-nucleus interactions. Thus the understanding of this broadening in the "cold" nuclear medium provides precious information for the interpretation of such high-energy processes [12,13].

An indication of nuclear broadening of the transverse momentum distribution for charged hadrons in muon and neutrino DIS was reported previously $[3,14]$. This Letter presents the first detailed measurement of the $p_{t}$-broadening for identified mesons and various nuclei as a function of the relevant kinematic variables.

The transverse hadron momentum $p_{t}$ is defined relative to the direction of the virtual photon, which is determined by the kinematics of the incident and the scattered lepton. The $p_{t}$-broadening $\Delta\left\langle p_{t}^{2}\right\rangle_{A}^{h}$ is defined as the difference of the average squared transverse momentum of the detected hadron of type $h$ produced on a nuclear target with atomic mass number $A$ and that on a deuterium (D) target: 
Table 1

Accumulated yield of selected hadrons for the various nuclear targets.

\begin{tabular}{lccc}
\hline Target & $\pi^{+} \times 10^{3}$ & $\pi^{-} \times 10^{3}$ & $K^{+} \times 10^{3}$ \\
\hline $\mathrm{D}$ & 1781 & 1445 & 356 \\
$\mathrm{He}$ & 134 & 107 & 27 \\
$\mathrm{Ne}$ & 380 & 303 & 82 \\
$\mathrm{Kr}$ & 321 & 260 & 72 \\
$\mathrm{Xe}$ & 193 & 157 & 44 \\
\hline
\end{tabular}

$\Delta\left\langle p_{t}^{2}\right\rangle_{A}^{h}=\left\langle p_{t}^{2}\right\rangle_{A}^{h}-\left\langle p_{t}^{2}\right\rangle_{\mathrm{D}}^{h}$

The broadening may depend on the hadronic variable $z$, and on the leptonic variables $v, Q^{2}$, and $x$, with $x=\frac{Q^{2}}{2 M v}$ the Bjorken variable and $M$ the nucleon mass.

The measurements were performed at Hermes using the 27.6 $\mathrm{GeV}$ lepton $\left(e^{+}\right.$or $\left.e^{-}\right)$beam stored in the HERA ring at DEsY. The targets consisted of polarized or unpolarized D, unpolarized $\mathrm{He}$, $\mathrm{Ne}, \mathrm{Kr}$, or Xe gas in natural abundance. The target atoms were injected into a $40 \mathrm{~cm}$ long, open-ended tubular storage cell through which the lepton beam passed. Target areal densities higher than $10^{16}$ nucleons $/ \mathrm{cm}^{2}$ were obtained for unpolarized gases and of about $2 \times 10^{14}$ nucleons $/ \mathrm{cm}^{2}$ for polarized D. The scattered lepton and the produced hadrons were detected in coincidence by the HeRmes spectrometer [15]. Leptons were distinguished from hadrons with an average efficiency of $99 \%$ and a contamination level of less than $1 \%$ by using a transitionradiation detector, a scintillator pre-shower counter, a dual-radiator ring-imaging Čerenkov (RICH) detector, and an electromagnetic calorimeter. Scattered leptons were selected by imposing the constraints $Q^{2}>1 \mathrm{GeV}^{2}, W^{2}>10 \mathrm{GeV}^{2}$, and $y<0.85$, where $W^{2}$ is the squared invariant mass of the photon-nucleon system and $y=v / E$ the energy fraction of the virtual photon in the target rest frame, with $E$ being the beam energy. All hadrons detected per event were considered independently in the analysis. In order to suppress the contributions from target remnant fragmentation, the requirement $z>0.2$ was imposed.

The numbers of identified and selected charged pions and positive kaons are given in Table 1. Negative kaons and antiprotons are not considered in this analysis due to low statistical precision. Protons were not considered because they are not well enough described by the Monte Carlo simulation used for the unfolding procedure described below.

The data used for the analysis of $p_{t}$-broadening were corrected for QED radiative effects, instrumental resolution, and acceptance. This was achieved using a Monte Carlo simulation based unfolding procedure [16], which accounted for event migration between different kinematic bins. The PYTHIA event generator [17], in conjunction with a special set of JETSET fragmentation parameters [18], was used together with the RADGEN [19] simulation of QED radiative effects and with a GEANT [20] based simulation of the detector. The possible dependence of the unfolding procedure on the target type used in the Monte Carlo simulation was studied with a special version of the LEPTO [21] generator and JETSET where the nuclear form factors and Fermi motion for D and Xe were incorporated. The results for both targets were found to be consistent. Therefore, a Monte Carlo simulation for the D target was used to correct the results for all targets.

Selected events were binned in $p_{t}^{2}$ and in either $z, v, Q^{2}$, or $x$. These two-dimensional ${ }^{2}$ distributions were unfolded using smearing matrices that hold information about migration among

\footnotetext{
2 A two-dimensional unfolding still leaves room for acceptance effects if both the yield and the magnitude of the effect depends non-linearly on the variables that are not included in the two-dimensional binning. An unfolding in more than two dimensions was not feasible in this analysis.
}

Table 2

Average kinematics for the $\left(\pi^{+}\right) p_{t}$-broadening results. The $v, Q^{2}, x$, and $z$ kinematics are for the Xe target.

\begin{tabular}{|c|c|c|c|c|}
\hline & $\langle\nu\rangle[\mathrm{GeV}]$ & $\left\langle Q^{2}\right\rangle\left[\mathrm{GeV}^{2}\right]$ & $\langle x\rangle$ & $\langle z\rangle$ \\
\hline \multicolumn{5}{|c|}{$\Delta\left\langle p_{t}^{2}\right\rangle$ vs. $A$} \\
\hline He & 14 & 2.4 & 0.10 & 0.42 \\
\hline $\mathrm{Ne}$ & 14 & 2.4 & 0.10 & 0.42 \\
\hline $\mathrm{Kr}$ & 14 & 2.4 & 0.10 & 0.41 \\
\hline $\mathrm{Xe}$ & 14 & 2.4 & 0.10 & 0.41 \\
\hline \multicolumn{5}{|l|}{$\Delta\left\langle p_{t}^{2}\right\rangle$ vs. $v$} \\
\hline$\nu$-bin\# 1 & 8 & 2.1 & 0.14 & 0.49 \\
\hline$v$-bin\# 2 & 12 & 2.5 & 0.11 & 0.43 \\
\hline$v$-bin\# 3 & 15 & 2.6 & 0.10 & 0.40 \\
\hline$\nu$-bin\# 4 & 19 & 2.4 & 0.07 & 0.37 \\
\hline \multicolumn{5}{|c|}{$\Delta\left\langle p_{t}^{2}\right\rangle$ vs. $Q^{2}$} \\
\hline$Q^{2}$-bin\# 1 & 14 & 1.4 & 0.06 & 0.42 \\
\hline$Q^{2}$-bin\# 2 & 14 & 2.5 & 0.10 & 0.41 \\
\hline$Q^{2}$-bin\# 3 & 14 & 3.9 & 0.15 & 0.40 \\
\hline$Q^{2}$-bin\# 4 & 15 & 6.5 & 0.25 & 0.39 \\
\hline \multicolumn{5}{|l|}{$\Delta\left\langle p_{t}^{2}\right\rangle$ vs. $x$} \\
\hline$x$-bin\# 1 & 15 & 1.6 & 0.06 & 0.40 \\
\hline$x$-bin\# 2 & 12 & 3.0 & 0.13 & 0.42 \\
\hline$x$-bin\# 3 & 12 & 5.5 & 0.25 & 0.42 \\
\hline$x$-bin\# 4 & 10 & 8.1 & 0.42 & 0.41 \\
\hline \multicolumn{5}{|l|}{$\Delta\left\langle p_{t}^{2}\right\rangle$ vs. $z$} \\
\hline z-bin\# 1 & 15 & 2.4 & 0.10 & 0.32 \\
\hline$z$-bin\# 2 & 13 & 2.4 & 0.11 & 0.53 \\
\hline$z$-bin\# 3 & 12 & 2.4 & 0.11 & 0.75 \\
\hline$z$-bin\# 4 & 11 & 2.3 & 0.12 & 0.94 \\
\hline
\end{tabular}

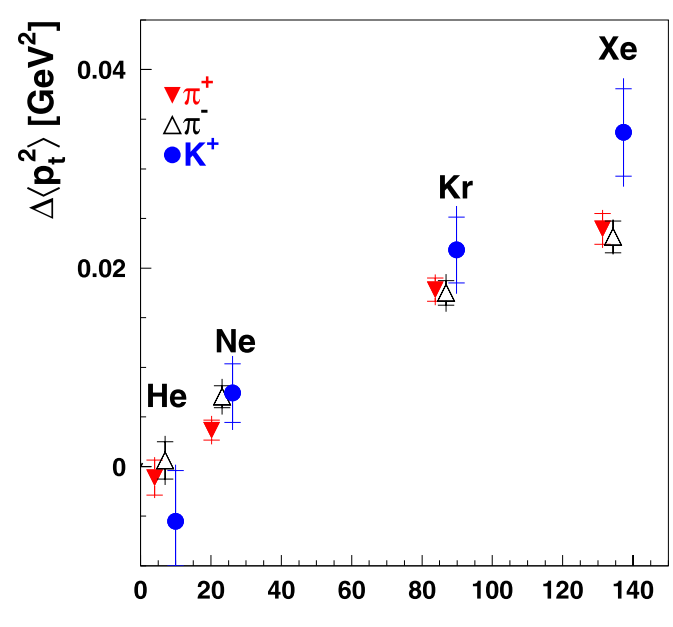

A

Fig. 1. The $p_{t}$-broadening for $\pi^{+}, \pi^{-}$, and $K^{+}$mesons as a function of atomic mass number $\mathrm{A}$. The inner error bars represent the statistical uncertainties; the total error bars represent the total uncertainty, obtained by adding statistical and systematic uncertainties in quadrature.

kinematic bins. This method inflates the statistical errors to account for this migration [22]. The impact of the unfolding on $\left\langle p_{t}^{2}\right\rangle$ ranges from $60 \%$ to almost zero with increasing $v$, and is about $20 \%$ for all $z$ bins. It varies from $20 \%$ to $30 \%$ with increasing $Q^{2}$, and is between $15 \%$ and $40 \%$ for the $x$-bins. After this correction, the average $p_{t}^{2}$ value was calculated in each bin of $z, v, x$, or $Q^{2}$ and then $\left\langle p_{t}^{2}\right\rangle_{\mathrm{D}}$ was subtracted from $\left\langle p_{t}^{2}\right\rangle_{A}$. A systematic uncertainty due to the unfolding procedure was estimated varying the background models for contributions originating from outside the spectrometer acceptance. This uncertainty is the dominant contribution to the total systematic uncertainty. 


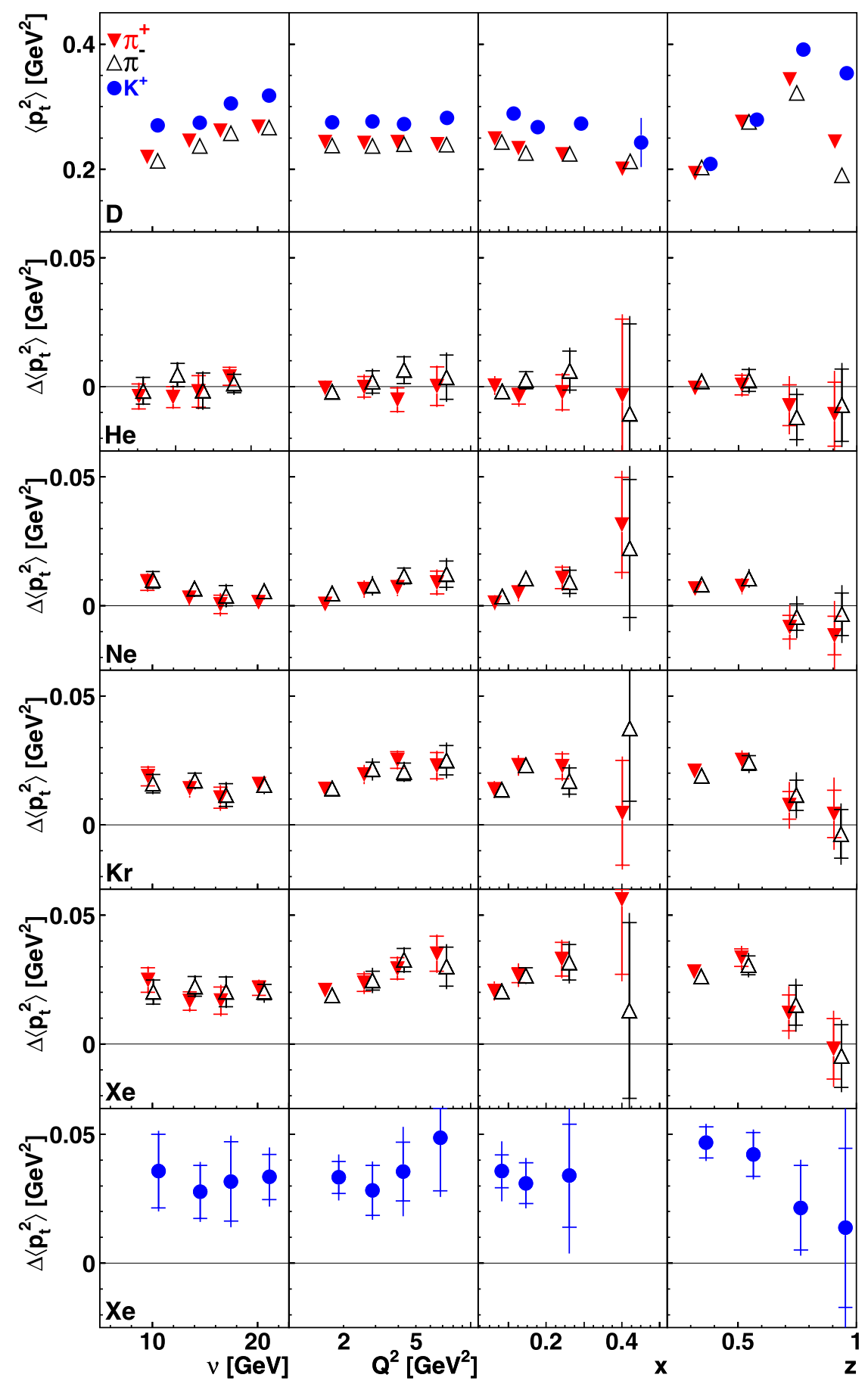

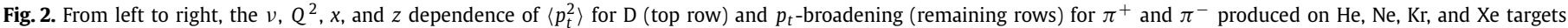

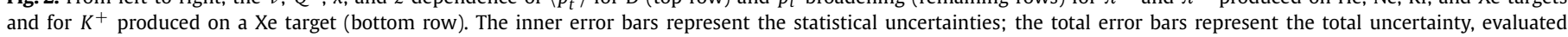
as the sum in quadrature of statistical and systematic uncertainties.

The charged pion yields were corrected for pions from the decay of exclusively-produced $\rho^{0}$ mesons. A correction for such pions, where at least one decay pion was in the acceptance, was made using a special Monte Carlo generator [23] that was tuned to HERMES data to obtain the correct relative contribution from coherent and incoherent $\rho^{0}$ production [24]. This Monte Carlo generator also simulated the measured kinematic dependence of coherent $\rho^{0}$ production from heavier targets. The exclusive $\rho^{0}$ correction was found to be less than $1 \%$ over the whole kinematic range, except at the highest $z$-value where it was about $10 \%$. The contribution of vector-meson decay to the kaon sample was found to be negligible and no correction was included for this. A systematic uncertainty due to Rich hadron misidentification was estimated to be smaller than $1 \%$.

The $p_{t}$-broadening for $\pi^{+}, \pi^{-}$, and $K^{+}$was determined for four nuclei as a function of either $v, Q^{2}, x$, or $z$, while integrating over the other kinematic variables. The average kinematics of the $p_{t}$-broadening results are shown in Table 2 . The results reveal negligible or small correlations among $z$ and the other variables while the average values of $x$ and $Q^{2}$ and those for $1 / v$ and $x\left(Q^{2}\right)$ are 
highly correlated due to the fixed target kinematics and the forward acceptance of the HERMES spectrometer. Note, however, that for the first (second) column $\left\langle Q^{2}\right\rangle(\langle v\rangle)$ is essentially constant over the whole measured $v\left(Q^{2}\right)$ range as shown in Table 2 .

The nuclear mass dependence of the $p_{t}$-broadening is presented in Fig. 1. The broadening increases with mass number $A$. It is similar for the charged pions and seems to be systematically higher for positively charged kaons. The precision of the data does not allow a firm conclusion about the functional form of the increase of the data with $A$. There is no clear indication of a saturation of the $p_{t}$-broadening at large atomic mass numbers, supporting models which treat its origin in the partonic stage. Within such models, this behavior suggests that the color neutralization happens near the surface of the nucleus or outside for the average kinematics of this measurement [25].

The panels presented in Fig. 2 show $\left\langle p_{t}^{2}\right\rangle$ for D (top row) and the $p_{t}$-broadening (remaining rows) as a function of either $v, Q^{2}$, $x$, and $z$ for $\pi^{+}$or $\pi^{-}$for the various nuclear targets. Since the uncertainties of the $K^{+}$sample are rather large, only the results for the Xe target are presented in the bottom row. The values of $\left\langle p_{t}^{2}\right\rangle$ for $\mathrm{D}$ are between 0.2 and $0.4 \mathrm{GeV}^{2}$ while the $p_{t}$-broadening shows values from 0 up to about $0.05 \mathrm{GeV}^{2}$. For Xe the $p_{t}$-broadening adds in average about $10 \%$ to $15 \%$ to $\left\langle p_{t}^{2}\right\rangle$.

The data do not reveal a significant dependence on $v$ in the kinematic range covered. Since models that describe hadron formation in nuclei commonly connect formation length with $v$, the basically flat behavior in $v$ supports again the picture that color neutralization mainly happens at the surface (or outside) of the nucleus for the HeRMes kinematics [25]. The effect slightly increases with $Q^{2}$ in contrast to the model calculation in Ref. [26], where a decrease of the broadening with $Q^{2}$ is predicted, and in agreement with the model calculation in Ref. [27]. The behavior as a function of $x$ is very similar to the $Q^{2}$ behavior, due to a strong correlation between $x$ and $Q^{2}$ in the Hermes kinematics, hence it cannot be excluded that the $Q^{2}$ dependence observed is actually an underlying $x$ dependence or both a $Q^{2}$ and $x$ dependence. The statistical precision of the data presented here does not allow the study of the $Q^{2}$ and $x$ dependence separately, or any other two kinematic observables.

The $p_{t}$-broadening is seen to vanish as $z$ approaches unity while the $\left\langle p_{t}^{2}\right\rangle$ for $\mathrm{D}$ is 0.2 or higher in the highest $z$-bin. Due to energy conservation the struck quark cannot have lost energy when $z=1$, leaving no room for broadening apart from a possible modification of the primordial quark transverse momentum. The observed vanishing of the $\Delta\left\langle p_{t}^{2}\right\rangle_{A}^{h}$ at high values of $z$ indicates that there is no or little dependence of the primordial transverse momentum on the size of the nucleus. It also indicates that $p_{t}$-broadening is not due to elastic scattering of pre-hadrons or hadrons already produced within the nuclear volume, as this would lead to substantial broadening even for values of $z$ very close to unity.

Detailed Monte Carlo studies have been performed comparing the measured $p_{t}^{2}$ distributions with model assumptions. These studies indicate that the whole $p_{t}$ range contributes to the broadening and that broadening increases linearly with $p_{t}$.

In summary, the first detailed determination of $p_{t}$-broadening in semi-inclusive deep-inelastic scattering for charged pions and positively-charged kaons was performed on $\mathrm{He}, \mathrm{Ne}, \mathrm{Kr}$, and Xe targets. The broadening was measured as a function of the atomic number $A$ and the kinematic variables $v, Q^{2}, x$ or $z$. The broadening increases with $A$ and remains constant with $v$, suggesting that the effect is due to the "partonic" stage and that color neutralization happens near the surface or outside the nucleus. The effect increases with increasing $Q^{2}$ and $x$, and vanishes as $z$ approaches unity.

\section{Acknowledgements}

We thank A. Accardi, B.Z. Kopeliovich, H.J. Pirner, and X.N. Wang for interesting and useful discussions. We gratefully acknowledge the DesY management for its support, the staff at DESY and the collaborating institutions for their significant effort, and our national funding agencies for financial support.

\section{References}

[1] D. de Florian, R. Sassot, M. Stratmann, Phys. Rev. D 75 (2007) 114010; D. de Florian, R. Sassot, M. Stratmann, Phys. Rev. D 76 (2007) 074033.

[2] L. Osborne, et al., Phys. Rev. Lett. 40 (1978) 1624.

[3] J. Ashman, et al., Z. Phys. C 52 (1991) 1.

[4] M. Adams, et al., Phys. Rev. D 50 (1994) 1836

[5] A. Airapetian, et al., Eur. Phys. J. C 20 (2001) 479;

A. Airapetian, et al., Phys. Lett. B 577 (2003) 37;

A. Airapetian, et al., Phys. Rev. Lett. 96 (2006) 162301

[6] A. Airapetian, et al., Nucl. Phys. B 780 (2007) 1.

[7] B. Andersson, et al., Phys. Rep. 97 (1983) 31;

B. Andersson, The Lund Model, Cambridge University Press, 1998.

[8] A. Bialas, M. Gyulassy, Nucl. Phys. B 291 (1987) 793.

[9] B. Kopeliovich, Phys. Lett. B 243 (1990) 141.

[10] B.Z. Kopeliovich, et al., Nucl. Phys. A 782 (2007) 224.

[11] R. Baier, et al., Nucl. Phys. B 484 (1997) 265.

[12] R. Baier, et al., Ann. Rev. Nucl. Part. Sci. 50 (2000) 37.

[13] A. Accardi, Phys. Rev. C 76 (2007) 034902.

[14] N.M. Agababyan, et al., Phys. Atom. Nucl. 66 (2003) 1310.

[15] K. Ackerstaff, et al., Nucl. Instr. Meth. A 417 (1998) 230.

[16] A. Airapetian, et al., Phys. Rev. D 75 (2007) 012007.

[17] T. Sjöstrand, et al., Comput. Phys. Commun. 135 (2001) 238.

[18] B. Maiheu, PhD thesis, University of Gent, 2006; A. Hillenbrand, PhD thesis, University of Erlangen, DESY-THESIS-2005-035, 2005.

[19] I. Akushevich, H. Böttcher, D. Ryckbosch, hep-ph/9906408.

[20] R. Brun, R. Hagelberg, M. Hansroul, J. Lassalle, CERN Report CERN-DD-78-2-REV, 1978.

[21] G. Ingelman, A. Edin, J. Rathsman, Comput. Phys. Commun. 101 (1997) 108.

[22] A. Airapetian, et al., Phys. Rev. D 71 (2005) 012003.

[23] A. Airapetian, et al., Eur. Phys. J. C 17 (2000) 3898.

[24] Y. Van Haarlem, PhD thesis, University of Gent, DESY-THESIS-2007-033, 2007.

[25] A. Accardi, arXiv:0808.0656 [nucl-th].

[26] B.Z. Kopeliovich, J. Nemchik, E. Predazzi, A. Hayashigaki, Nucl. Phys. A 740 (2003) 211.

[27] S. Domdey, D. Grünewald, B.Z. Kopeliovich, H.J. Pirner, Nucl. Phys. A 825 (2009) 200. 